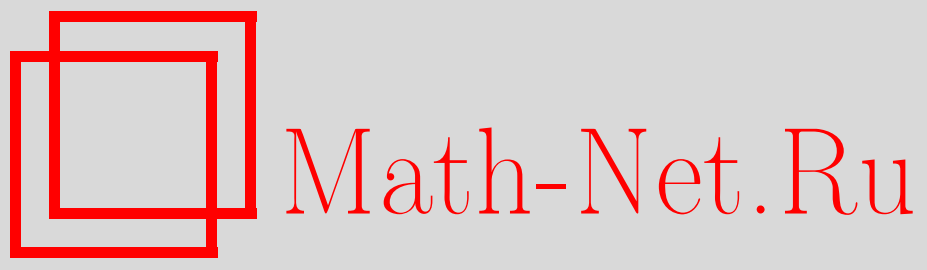

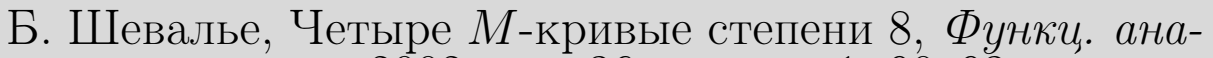
лиз и его прил., 2002, том 36, выпуск 1, 90-93

DOI: https://doi.org/10.4213/faa184

Использование Общероссийского математического портала Math-Net.Ru подразумевает, что вы прочитали и согласны с пользовательским соглашением

http://www . mathnet.ru/rus/agreement

Параметры загрузки:

IP : 44.207 .124 .84

26 апреля 2023 г., 14:18:43

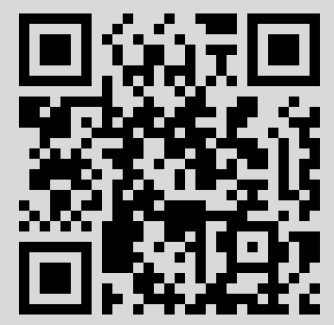


mult $_{z} Z=4-$ противоречие. Это завершает проверку условий (i)-(iii) и доказывает теорему. Детали намеченных рассуждений будут опубликованы в другой работе.

\section{ЛИТЕРАТУРА}

1. Манин Ю. И. Матем. сб., 72, №2, 161-192 (1967). 2. Исковских В. А., Манин Ю. И. Матем. сб., 86, № 1, 140-166 (1971). 3. Пухликов А. В. Функц. анализ и его прил., 33, вып. 4, 88-90 (1999). 4. Пухликов А. В. Матем. сб., 191, №6, 101-126 (2000). 5. Пухликов А. В. Изв. РАН., сер. матем., 64, 3, 131-150 (2000).

Институт системного анализа РАН

Поступило в редакцию 26 декабря 2000 г.

УДК 512.77

\section{Четыре $M$-кривые степени 8}

(C) 2002. Б. ШЕВАЛЬЕ

Мы доказываем существование четырех топологических типов пар $\left(\mathbb{R} \mathbb{P}^{2}, C_{8}\right)$ (изотопических типов $C_{8}$ в $\mathbb{R} \mathbb{P}^{2}$ ), где $C_{d}$ обозначает гладкую вещественную алгебраическую плоскую кривую степени $d$. Классификация пар $\left(\mathbb{R} \mathbb{P}^{2}, C_{d}\right)$, составляющая часть 16 -й проблемы Гильберта, до сих пор далека от завершения. См. обзоры $[2,6,7]$, а также обзор [3], где приведен список из 78 (соответственно 13) изотопических типов, про которые было известно, что они реализуемы (соответственно неизвестно, реализуемы ли) $M$-кривыми $C_{8}$ (кривая $C_{d}$ называется $M$-кривой, если она имеет максимально возможное число овалов, которое равно 22 при $d=8$ ). Теорема 1 заменяет эти числа на 82 (соответственно 9). Основное утверждение, используемое при построениях с контролируемой топологией, - это теорема Виро о склеивании карт $[8,9]$ (см. также $[4,1])$.

Теорема 1. Следующие изотопические типь ${ }^{1)}$ реализуемы вещественными алгебрачческими кривыми степени 8:

$$
16 \sqcup 1\langle 2 \sqcup 1\langle 2\rangle\rangle, \quad 2 \sqcup 1\langle 2 \sqcup 1\langle 16\rangle\rangle, \quad 13 \sqcup 1\langle 2 \sqcup 1\langle 5\rangle\rangle, \quad 5 \sqcup 1\langle 2 \sqcup 1\langle 13\rangle\rangle .
$$

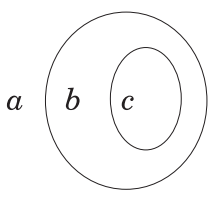

Рис. 1

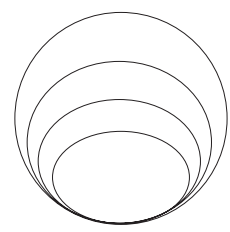

Рис. 2

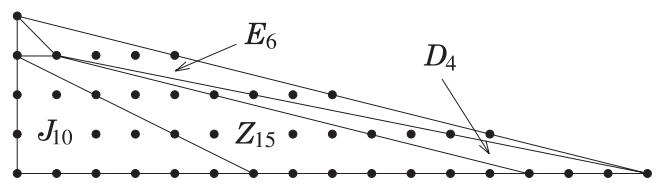

Pис. 3

Изотопические типы теоремы 1 реализуются возмущениями кривой $C_{8}^{\text {sing }}=$ $\{(X: Y: Z) \mid F=0\}, F=\prod_{k=1}^{4}\left(Y Z-a_{k} Y^{2}-X^{2}\right), a_{1}<\cdots<a_{4}$. Это объединение

1) Мы используем обозначения Виро [7]: $a \sqcup 1\langle b \sqcup 1\langle c\rangle\rangle$ кодирует изотопический тип кривой на рис. 1, где через $a, b$ и $c$ обозначены числа овалов, лежащих вне друг друга в соответствующей области. 
четырех коник, имеющих попарные пересечения кратности 4 в точке $(0: 0: 1)$. Такая кривая не удовлетворяет условиям теоремы Виро о склеивании. При этом $k$-я коника имеет или не имеет вещественные пересечения с прямой $Z=0$ в зависимости от знака числа $a_{k}$, но при любых знаках $C_{8}^{\text {sing }}$ расположена в некоторой аффинной карте так, как на рис. 2.

Введем аффинные координаты $x=X / Z, y=Y / Z$ и $u=x, v=y-x^{2}$. В этих координатах $C_{8}^{\text {sing }}$ имеет вид $\{f(x, y)=0\}$ и $\{\tilde{f}(u, v)=0\}$ соответственно, где $f(x, y)=F(x, y, 1)$ и $\tilde{f}(u, v)=f\left(u, v+u^{2}\right)=\prod_{k=1}^{4}\left(v-a_{k}\left(v+u^{2}\right)^{2}\right)=$ $\prod_{k=1}^{4}\left(v-a_{k} u^{4}\right)+\ldots$ Таким образом, $C_{8}^{\text {sing }}$ имеет особенность в $(0: 0: 1)$, которая является полуквазиоднородной относительно координат $(u, v)$ (см. [6]). Эту особенность можно сгладить методом Виро с помощью карты, которая получается склеиванием меньших карт, как изображено на рис. 4 и 5 (в этот момент мы выбираем параметры $\left.a_{1}, \ldots, a_{4}\right)$. Эти карты соответствуют разбиению треугольника $[(0,0),(16,0),(0,4)]$, изображенному на рис. 3 ; они получаются бирациональными преобразованиями из карт распусканий особенностей $E_{6}, D_{4}, J_{10}$ и $Z_{15}$ (см. [6, пп. 4.1, 4.2, 4.4]). Рисунок 4 дает изотопические типы $16 \sqcup 1\langle 2 \sqcup 1\langle 2\rangle\rangle$ и $2 \sqcup 1\langle 2 \sqcup 1\langle 16\rangle\rangle$; рис. 5 дает изотопические типы $13 \sqcup 1\langle 2 \sqcup 1\langle 5\rangle\rangle$ и $5 \sqcup 1\langle 2 \sqcup 1\langle 13\rangle\rangle$. Чтобы поменять местами количества внутренних и внешних овалов (т.е. $13 \leftrightarrow 5$ и $16 \leftrightarrow 2$ ), достаточно отразить рис. 4 и 5 относительно горизонтальной оси (это соответствует замене $a_{k}$ на $-a_{k}$ ).

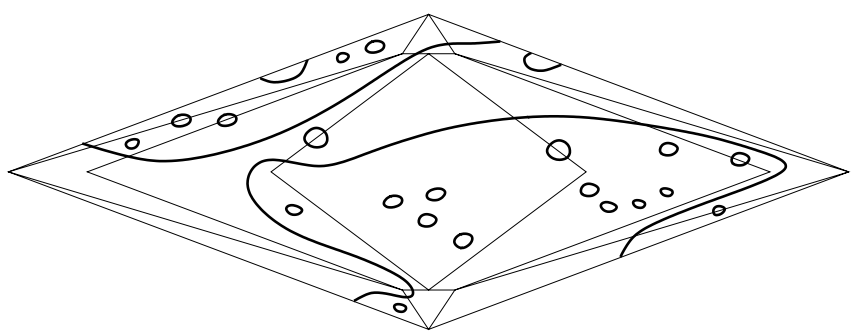

Рис. 4

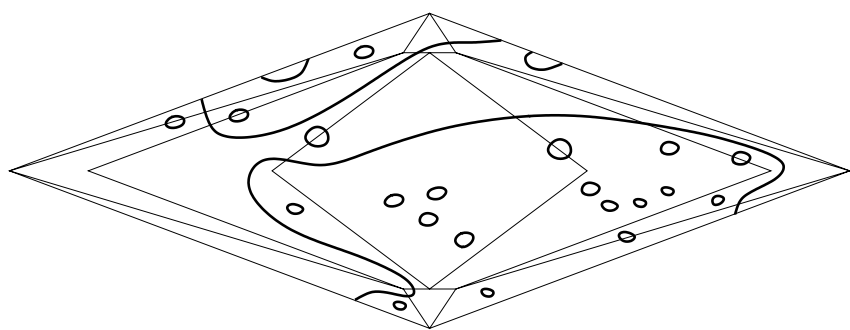

Рис. 5

Такое сглаживание может быть выполнено в координатах $(u, v)$. Возвращаясь к координатам $(x, y)$, мы получим мономы степени, большей чем 8 , но, согласно [5, Lemma 12], можно осуществить топологически эквивалентное сглаживание при помощи полинома от $x, y$ степени 8 . Это завершает доказательство теоремы 1. 
Доказательства в [5] основаны на теореме Римана-Роха. Здесь мы приводим прямое полное доказательство для нужного нам частного случая.

Фиксируем полином $\tilde{g}(u, v)=\sum_{i+4 j \leqslant 16} b_{i j} u^{i} v^{j}$, реализующий одну из четырех рассматриваемых карт (здесь и далее все индексы предполагаются неотрицательными). Обозначим через $\tilde{g}_{t}$ полином Виро: $\tilde{g}_{t}(u, v)=\sum_{i+4 j \leqslant 16} b_{i j}(t) u^{i} v^{j}$, где $b_{i j}(t)=t^{16-(i+4 j)} b_{i j}$. Выберем $a_{1}, \ldots, a_{4}$ так, чтобы совпали ограничения полиномов $\tilde{f}$ и $\tilde{g}$ на отрезок $[(16,0),(0,4)]$, т. е. чтобы коэффициенты при мономах $u^{16-4 j} v^{j}, j=0, \ldots, 4$, в $\tilde{f}$ были бы такими же, как и в $\tilde{g}$.

Рассмотрим полином $h(x, y)=\sum_{r+s \leqslant 8} \varepsilon_{r s} x^{r} y^{s}$ с неопределенными коэффициентами. В координатах $(u, v)$ он принимает вид $\tilde{h}(u, v):=h\left(u, v+u^{2}\right)=$ $\sum_{i+2 j \leqslant 16} \eta_{i j}(\vec{\varepsilon}) u^{i} v^{j}$, где через $\vec{\varepsilon}=\left(\varepsilon_{00}, \varepsilon_{01}, \ldots\right)$ обозначен вектор, составленный из всех $\varepsilon_{r s}(r+s \leqslant 8)$, и $\eta_{i j}(\vec{\varepsilon})$ - некоторые явно выписываемые линейные функции от $\vec{\varepsilon}$. Обозначим через $\vec{\varepsilon}(t)$ решение системы линейных уравнений

$$
\begin{cases}\eta_{i j}(\vec{\varepsilon})=b_{i j}(t), & i+4 j<16, \\ \eta_{i j}(\vec{\varepsilon})=0, & i+4 j=16 .\end{cases}
$$

Фактически это 17 независимых систем: для каждого $m=0, \ldots, 16$ мы имеем систему уравнений, отвечающую множеству индексов $\{(i, j) \mid i+2 j=m\}$. Легко убедиться в том, что матрицы всех этих систем треугольны и невырожденны. Поэтому решение существует и его можно явно выразить через $b_{i j}(t)$. Например, $\varepsilon_{r s}(t)$ при $r+2 s \leqslant 8-$ это просто коэффициент при $x^{r} y^{s}$ в полиноме $g(x, y):=$ $\tilde{g}\left(x, y-x^{2}\right)$.

Положим $f_{t}(x, y)=f(x, y)+h_{t}(x, y)$, где $h_{t}(x, y)$ получено из $h(x, y)$ подстановкой $\vec{\varepsilon}=\vec{\varepsilon}(t)$. Полином $f_{t}(x, y)$, записанный в координатах $(u, v)$, принимает вид

$$
\tilde{f}_{t}(u, v)=\tilde{f}(u, v)+\sum_{i+4 j<16} b_{i j}(t) u^{i} v^{j}+\sum_{i+4 j>16} c_{i j}(t) u^{i} v^{j}, \quad \lim _{t \rightarrow 0} c_{i j}(t)=0 .
$$

Значит, используя такие же рассуждения, как и при доказательстве теоремы Виро (см. $[8,9])$, можно показать, что существует $t_{0}>0$, такое, что при $t \in\left(0, t_{0}\right]$ кривая $\left\{f_{t}=0\right\}$ получается из $\{f=0\}$ вклеиванием фрагмента $\{g=0\}$ в особую точку $(0: 0: 1)$.

ЗАмЕчАНИЕ. Этот подход можно рекурсивно применять к промежуточным картам, используемым при построении кривой $\{\tilde{g}=0\}$. Почти все изотопические типы $M$-кривых $C_{8}$ можно получить этим способом (слово «почти» порождает вопросы). Его также можно применять к гиперповерхностям.

Благодарности. Интерес к рассмотрению кривой $C_{8}^{\text {sing }}$ у меня возник после обсуждений с А. Б. Корчагиным. Я благодарен С. Ю. Оревкову за значительное улучшение изложения.

\section{ЛИТЕРАТУРА}

1. Gelfand I. M., Kapranov M. M., Zelevinsky A. V. Discriminants, Resultants, and Multidimensional Determinants. Birkhäuser, Boston, 1994. 2. Гудков Д. А. УМН, 29, 1-79 (1974). 3. Korchagin A. B. In: Visiting Scholars' Lectures-1997. Math. Series, Vol. 19, Texas Tech University, 1997, pp. 85-140. 4. Risler J.-J. Constructions d'hypersurfaces 
algébriques réelles (d'après Viro). Séminaire Bourbaki, Vol. 1992/93. Astérisque, No. 216 (1999), Exp. 763, pp. 69-86. 5. Shustin E. I. Алгебра и анализ, 10, 221-249 (1999). 6. Buро О. Я. Алгебра и анализ, 1, вып. 5, 1-73 (1989). 7. Виро О. Я. УМН, 41, 55-82 (1986). 8. Viro O. Ya. In: Lecture Notes in Math., Vol. 1960, 1984, pp. 187-200. 9. Вupo О. Я. Beщественные алгебраические многообразия с предписанными топологическими свойствами. Диссертация д. ф.-м. н., Ленинград, 1983; http://www.math.uu.se/〜oleg

Université Toulouse II, Département de Mathématiques, Laboratoire E. Picard, CNRS UMR 5580

Поступило в редакцию 22 января 2001 г.

УДК 512

\title{
О значениях показателей роста групп с условием малого сокращения
}

\author{
(c) 2002. А. Г. ЭршлЕР (ДюьИнА)
}

Мы рассматриваем конечно порожденную группу $G$ с фиксированной системой образующих $S$. Пусть $d-$ словарная метрика, соответствующая системе $S$, $B_{G}(n)$ - шар $\{g \in G: d(g, e) \leqslant n\}$, а $v=v_{S}(G)$ обозначает (экспоненциальный) показатель роста

$$
v=v_{S}(G)=\lim \sqrt[n]{\# B_{G}(n)},
$$

где \# - мощность множества.

Группа $G$ имеет экспоненциальный рост, если для некоторой (и, следовательно, для любой) конечной системы образующих $S$ (экспоненциальный) показатель роста $v_{S}(G)$ больше 1 .

Пусть $k$ - количество образующих группы $G$. Выполнены очевидные оценки

$$
1 \leqslant v \leqslant 2 k-1
$$

причем равенство в последнем неравенстве достигается только тогда, когда группа свободна.

В общем случае мало что известно о значениях экспоненциальных показателей роста. Но, например, известно, что показатель роста любой гиперболической группы является целым алгебраическим числом $[4,5]$.

В этой заметке мы рассматриваем множество

$$
\Omega=\left\{v \in[1, \infty) \mid v=v_{S}(G) \text { для некоторых } G \text { и } S\right\} .
$$

Это множество всюду плотно в $[1, \infty)$ (см. $[2,6])$. В $[6]$ спрашивается, какую мощность имеет $\Omega$.

Следующая теорема отвечает на этот вопрос.

Теорема 1. Пусть $\Omega_{2}=\{v \in[1, \infty) \mid v=v(G)$ для 2-порожденной группь $G\}$. тогда $\# \Omega_{2}=2^{\aleph_{0}}$.

Эта теорема является следствием такого результата:

ПредлОЖЕНИЕ 1. Существует функция $E: \mathbb{N} \rightarrow \mathbb{N}$ со следующим свойством. Пусть $r_{i}=\left(a^{E(i)} b^{E(i)}\right)^{100}$. Для $J \subset \mathbb{N}$ положим

$$
G_{J}=\left\langle a, b \mid r_{j}=1, j \in J\right\rangle .
$$

Тогда если $I \neq J$, то $v_{\{a, b\}}\left(G_{I}\right) \neq v_{\{a, b\}}\left(G_{J}\right)$. 